$$
\text { Conf }-930159-57
$$

UCRL-JC-111288

PREPRINT

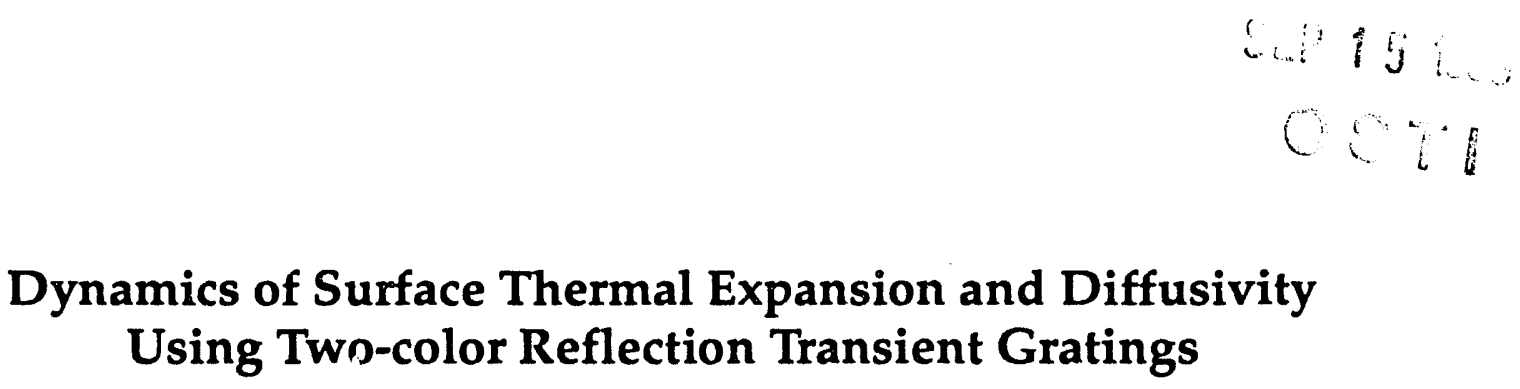

D. M. Pennington and C. B. Harris

This paper was prepared for submittal to the SPIE - The International Society of Optical Engineering Proceedings January 16-23, 1993

Los Angeles, CA

February 1993

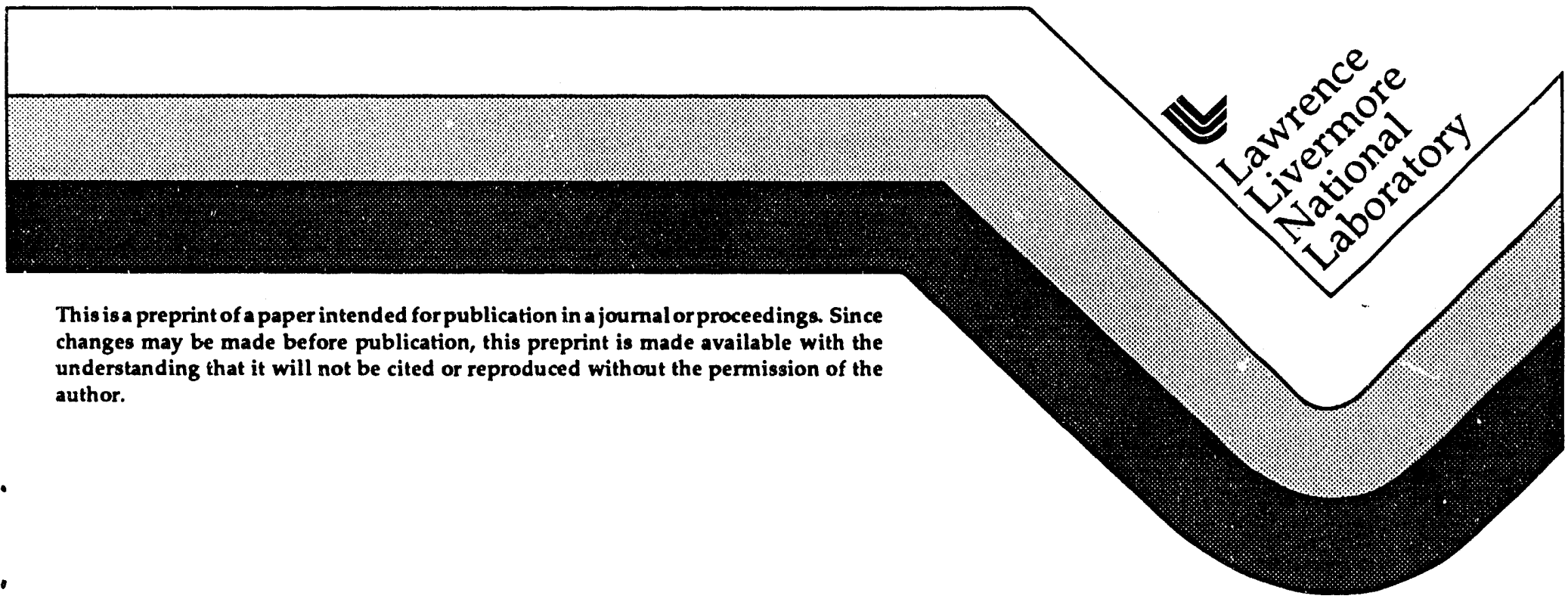




\section{DISCLAIMER}

This document was prepared as an account of work sponsored by an agency of the United States Governm . nt. Neither the United States Government nor the University of California nor any of their employees, makes any warranty, express or implied, or assumes any legal liability or responsibility for the accuracy, completeness, or usefulness of any information, apparat us, product, or process disclosed, or represents that its use would not infringe privately owned rights. Reference herein to any specific commercial products, process, or service by trade name, trademark, manufacturer, or otherwise, does not necessarily constitute or imply its endorsement, recommendation, or favoring by the United States Govermment or the University of California. The views and opinions of authors expressed herein do not necessarily state or reflect those of the United States Government or the University of California, and shall not be used for advertising or product endorsement purposes. 


\title{
Dynamics of Surface Thermal Expansion and Diffusivity Using Two-color Reflection Transient Gratings
}

\author{
D. M. Pennington ${ }^{*}$ and C. B. Harris \\ Department of Chemistry, University of Califormia, Berkeley, CA 94720 \\ * Present address: Lawrence Livermore National Laboratory, P.O. Box 808, Livermore. CA 94551
}

\begin{abstract}
We report ultrafast measurements of the dynamic thermal expansion of a surface and the temperature dependent surface thermal diffusivity using a two-color reflection transient grating technique. Studies were performed on p-type, $n$ type, and undoped $\mathrm{GaAs}(100)$ samples at several temperatures. Using a 75 fs ultraviolet probe with visible excitation beams, the electronic effects that dominate single color experiments become negligible; thus surface expansion due to heating and the subsequent contraction caused by cooling provide the dominant influence on the diffracted probe. The diffracted signal was composed of two components, thermal expansion of the surface and heat flow away from the surface, allowing the determination of the rate of expansion as well as the surface the val diffusivity. At room temperature a signal rise due to thermal expansion was observed, corresponding to a maximum dverage displacement of $\approx 1 \AA$ at 32 ps. Large fringe spacings were used, thus the dominant contributions to the signal were expansion and diffusion perpendicular to the surface. Values for the surface thermal diffusivity of $\mathrm{GaAs}$ were measured and found to be in reasonable agreement with bulk values above $50^{\circ} \mathrm{K}$. Below $50^{\circ} \mathrm{K}$, the diffusivity at the surface was more than an order of magnitude slower than in the bulk due to increased phonon boundary scattering. Comparison of the results with a straightforward thermal model yields good agreement over a range of temperatures $\left(12-300{ }^{\circ} \mathrm{K}\right)$. The applicability and advantages of the transient grating technique for studying photothermal and phoroacoustic phenomena are discussed.
\end{abstract}

\section{INTRODUCTION}

The interaction of laser radiation with matter is an extensively studied field, in particular, the response of a medium to the impulsive deposition of heat. ${ }^{1-7}$ A necessary consequence of this deposition is thermal expansion of the surface, and the simultaneous generation of acoustic waves through out the lattice. Thermal expansion of dielectric mirrors, propagation of heat in microelectronics, and the dissipation of heat during material processing serve as examples of the importance of this process. In particular, thermal expansion in semiconductors is a topic of technological as well as scientific importance since it plays an important role in determining the residual stresses in solid state devices. Lattic - mismatch and biaxial stress due to different rates of thermal expansion between multilayer semiconductor structures can generate dislocations that alter the band structure of the materials and influence carrier relaxation processes. Previous experiments have reported the observation of the transient perpendicular expansion of a heated surface, indirectly using picosecond photothermal deflection spectroscopy 8 , and directly using the two-color transient grating technique 9 . However, while photothermal deflection spectroscopy can only measure expansion perpendicular to the surface, the transient grating method has the potential for measurement of the expansion both in and out of the plane of the surface. In addition, the signal decay provides a measure of the thermal conductivity along each crystallographic axis. This paper discusses the use of a two-color reflection transient grating technique to measure the ultrafast expansion rate and thermal diffusivity of a surface.

In a transient grating experiment, two laser pulses with wave vectors $\dot{k}_{\text {exc }}^{\prime}$ and $k_{\text {exc }}^{\prime \prime}$ are temporally and spatially overlapped on the surface of a sample as shown in Fig. 1. The interference between the two pulses forms a sinusoidal grating pattern on the sample surface with a fringe spacing, $f$, related to the angle between the two beams, $\theta$, by the relation $f=\lambda_{\text {exc }} / 2 \sin (\theta / 2)$, where, $\lambda_{\text {exc }}$ is the excitation wavelength in vacuum. The optical energy absorbed by the material results in local heating and thermal expansion, simultaneously launching acoustic waves with a wavelength determined by the fringe spacing. This produces a time-dependent modulation of the sample density, resulting in a time-dependent oscillation of the real and imaginary indices of refraction with the same periodicity as the interference pattern. A variably time delayed probe pulse diffracts off the optically induced density modulations, producing a signal that reflects their periodicity, magnitude, and time dependence.
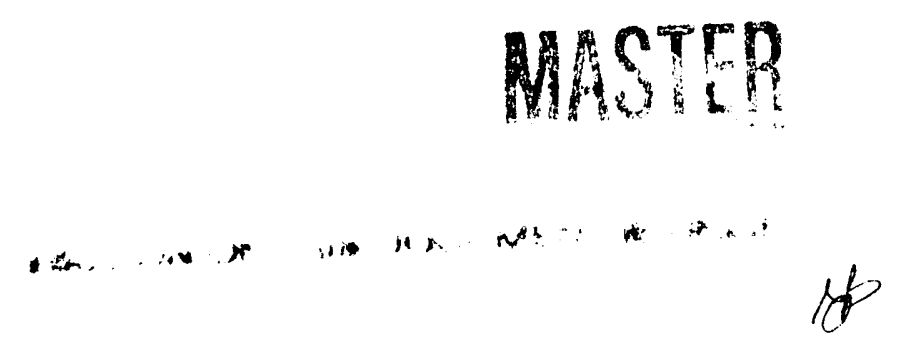
For many years transient gratings have been used to study a variety of ultrafast processes in condensed phase materials. ${ }^{10-14}$ In particular, the transient grating (TG) technique has proven quite successful for studying thermal flow 6,7 and acoustic propagation 15 in thin films as it has the advantage of non contact generation and detection of a well-defined heat source at the surface or in the bulk of a sample. Like the photothermal deflection method, the TG technique is sensitive to minute displacements of the surface, and can be used to determine the surface temperature profiles and expansion dynamics ${ }^{16}$. Unlike other photothermal methods, however, transient gratings have the ability to discriminate between signals arising from each of the three crystallographic axes of a sample simply by varying the periodicity of the grating and the excitation geometry. The results presented here were performed with a large fringe spacing, i.e., fringe spacing >> optical absorption depth. Under these conditions, the resulting signal is primarily due to expansion and contraction perpendicular to the surface region and can be modeled reasonably well with one-dimensional solutions. For small fringe spacing however, this technique is sensitive to the component of expansion in the plane of the surface, provided the thermal diffusivity is long relative to the rate of expansion.

While transient grating experiments have traditionally been performed in transmission, a recent paper by Fishman, et. al. has demonstrated that this method is sensitive to surface properties when performed in the reflection grating geometry, while transmission studies are dominated by bulk properties. ${ }^{17-19}$ This was experimentally demonstrated by using the technique to measure thermal flow and ultrasonic propagation in thin films. 6,7 The transmitted diffracted signal is proportional to the change in the dielectric constant, $\Delta \varepsilon$, created by the grating, which leads to a equal spatial weighting of the signal contributions throughout the material. The reflected signal, however, is proportional to $\frac{\mathrm{d} \Delta \varepsilon}{\mathrm{d} z}$ to first order in $\Delta \varepsilon$.

Thus the reflection signal is only generated from the spatial regions where an abrupt change of the dielectric constant exists, i.e., the surface or interfacial regions of the sample. For reflection transient gratings, the maximum depth from which signal can be effectively be generated is $\lambda / 4 \pi n$, which corresponds to a material with no absorption. 18 As the material becomes more optically dense (i.e., absorption depth $<$ optical wavelength), absorption becomes increasingly shallow, thus both the reflected signal and the transmitted signal are generated progressively closer to the surface. The surface can be selectively studied by usin ${ }_{0}$ a probe wavelength far down the edge of an intense absorption band where the optical density is low, thus creating a large difference between the spatial regions from which the transmitted and reflected signals are generated. As the probe wavelength approaches the absorption band, the difference in the spatial region sampled in reflection and transmission decreases. Thus by varying the laser wavelength and experimental grating geometry one can selectively probe to different depths in the sample, allowing the investigation of surface, interface. and bulk phenomena. Here we report the extension of this technique to the study of transient thermal expansion and diffusivity at a surface.

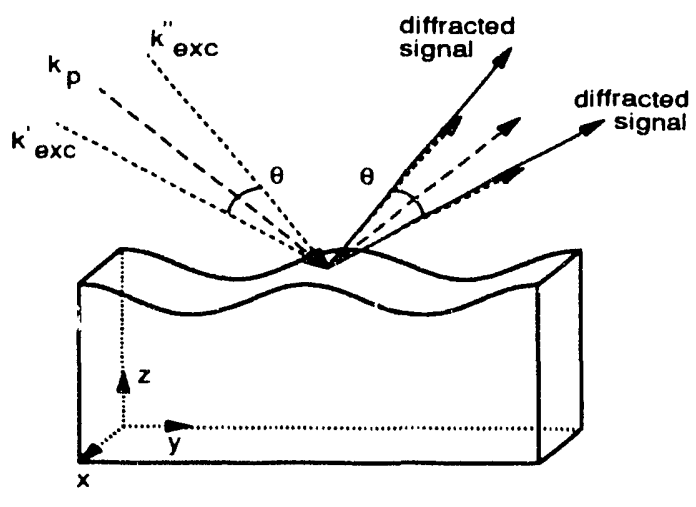

Fig. 1 Reflection transient grating geometry. Two grating excitation beams, with wave vectors $k_{e x c}^{\prime}$ and $k_{e x c}^{\prime \prime}$, cross at an angle $\theta$ at the surface. The time delayed probe beam bisects the angle between the excitation beams, giving rise to a diffracted signal collinear with both of the specularly reflected excitation beams.

\section{THEORY}

A complete theoretical treatment of the transient grating signal from highly absorbing materials in the reflection geometry has recently been published, providing the formalism for analyzing the data herein. ${ }^{17-19}$ This formalism includes the spatial distribution of the grating-induced dielectric constant profile perpendicular to the sample surface which had not previously been treated. If we consider a semi-infinite plane of absorbing material with a complex dielectric constant, $\varepsilon$, the reflected diffraction efficiency, $\eta$, is proportional to the squ'. modulus of the grating peak-to-null difference in the complex dielectric constant. For a sample with weak absorption, ne reflected diffracted signal arises from a maximum depth of 
$\lambda / 4 \pi n$, while the signal from a strongly absorbing sample is generated in a region within half an absorption depth of the surface or interface. A general expression for the one-dimensional reflected diffraction efficiency of the probe is given by

$$
\eta(z)=\frac{\omega^{2}}{c^{2}} \frac{\cos ^{2} \theta}{\left|\cos \theta+\sqrt{\varepsilon-\sin ^{2} \theta}\right|^{4}}\left|\int_{0}^{\infty} \mathrm{e}^{2 i \frac{\omega}{c} \sqrt{\tilde{\varepsilon}-\sin ^{2} \theta} z} \Delta \tilde{\varepsilon}(\mathrm{z}, \mathrm{t}) \mathrm{dz}\right|^{2},
$$

where $\varepsilon, c, \omega$, and $\Delta \varepsilon(z, t)$ are the unperturbed dielectric constant of the sample, speed of light, probe beam angular frequency, and grating peak-to-null modulation of the dielectric constant, respectively. The complex index of refraction, $\tilde{n}=(n+i k)=(\mathbb{E})^{1 / 2}$, is modulated with the grating periodicity, resulting in

$$
\tilde{n}^{\prime}(y, z, t)=\tilde{n}+\Delta \tilde{n}(y, z, t)=\tilde{n}+\cos (\beta y)\{\Delta n(z, t)+i \Delta k(z, t)\},
$$

where $\beta=4 \pi \sin (\theta) / \lambda_{\text {exc }}$ is the magnitude of the grating wave vector. Ignoring second order terms in $\Delta$ leads to a dielectric constant modulation with a grating peak-to-null amplitude of

$$
\Delta \tilde{\varepsilon}(z, t)=2 \tilde{n}\{\Delta n(z, t)+i \Delta k(z, t)\} .
$$

Thus in the sma!! angle limit, i.e. $|\mathcal{E}| \gg \sin ^{2} \theta$, Eqn. 1 can be reduced to

$$
\eta(z, t)=\frac{(4 \pi \tilde{n})^{2}}{|1+\tilde{n}|^{4}}\left|\int_{0}^{\infty} e^{2 i k_{z} z} \Delta \tilde{n}(z, t) d z\right|^{2}
$$

Here $\Delta \tilde{n}(z, t)$ is the grating peak-to-null modulation of the complex index of refraction, and $k_{z}$, which equals $\frac{2 \pi}{\lambda}(n+i k)$, is the mag,nitude of the complex probe wave vector, where $n$ and $k$ are the real and imaginary indices of refraction respectively.

The driving force for th transient grating signal in our experiment is the impulsive deposition of an amount of heat $q$ in the lattice by nonradiative relaxation following optical absorption. This leads to a spatially varying temperature distribution in the lattice given by

$$
\Delta T(x, y, z, l=0)=\frac{\Delta T_{o}}{2} \mathrm{e}^{-\alpha_{e x c^{z}}}(1+\cos (\beta y)) \mathrm{e}^{-\left(x^{2}+y^{2}\right) / 2 a^{2}} .
$$

Here $\Delta T_{o}=q\left(1-\mathrm{e}^{-\alpha_{e x c^{z}}}\right) / \rho C$ is the initial temperature rise at a grating peak a distance $z$ into the surface, $\alpha_{e x c}$ is the optical absorption coefficient at the excite wavelength, $\rho$ is the unperturbed density, $C$ is the heat capacity at constant volume, and $a$ is the excitation beam radius. Al time $t=0$, the sample has been excited by the driving force, but there are as yet no displacements, thus the total strain and the resulting stress must be zero. As the surface thermally expands, the grating peaks are displaced towards the grating nulls and perpendicular to the plane of the surface, and counter propagating acoustic waves are simultaneously launched. This creates a strain, $\Sigma$, in the medium which in turn induces an isotropic thermal stress given by $3 B \gamma \Delta T_{o}$, where $B$ is the bulk modulus and $\gamma$ is the coefficient of thermal expansion. Because excited states have a negligihle effect on the time scale of our signal, only strain induced contributions to $\Delta \tilde{n}$ need to be considered. The grating peak-to-null variations in the real and imaginary indices of refraction due to the presence of strain, respectively, are:

$$
\begin{aligned}
& \Delta n(z, t)=-\Sigma(z, t) \frac{\left(n^{2}-1\right)}{2 n} \text { and } \\
& \Delta k(z, t)=-\Sigma(z, t) k .
\end{aligned}
$$

The contributions to $\Delta n$ and $\Delta k$ have been discussed in detail previously. ${ }^{11}$ The strain is related to local changes in the material density by the relation

$$
\Delta \rho(z, t)=-\rho_{o} \Sigma(z, t) .
$$

The density change in one dimension is proportional to first order to the displacement, $\Delta u$, of the grating in that dimension, ${ }^{20}$ thus $\Delta \bar{n}(z, t) \propto \Delta \rho(z, t) \propto \Delta u(z, t)$. The displacement of the surface due to thermal expansion provides a real 
experimental observable which we can use in place of $\Delta \tilde{n}(z, t)$ in Eqn. 4 to calculate the diffraction efficiency of the reflected probe beam in the $z$-direction. To find the time-dependent expansion in the $z$-direction, the acoustic field equations must be solved. For an isotropic cubic medium such as GaAs, they need only be solved in one dimension. Thus the relevant equations are:

$$
\begin{aligned}
\Sigma_{z z} & =\frac{\partial u_{z}}{\partial z} \\
\rho \frac{\partial^{2} u_{z}}{\partial t^{2}} & =\frac{\partial \sigma_{z z}}{\partial z} \quad \text { and } \\
\sigma_{z z} & =\rho v_{s} B \Sigma_{z z}-3 B \gamma \Delta T_{o},
\end{aligned}
$$

where $u_{z}$ is the material displacement in the z-direction resulting from the strain, $\Sigma_{z z}$, and $v_{s}$ is the velocity of sound in the material. These equations have been solved with the initial condition of zero strain everywhere and the boundary condition of zero stress at the free surface, i.e. $\sigma_{z z}(z=0)=0$, at all times to find the temporal evolution of the strain field ${ }^{5}$ :

$$
\Sigma(z, t) \propto\left\{e^{-\alpha_{e x c^{z}}}\left(1-\frac{1}{2} e^{\left.-\alpha_{e x c^{v} s^{t}}\right)}\right\}-\left\{\frac{(1-R) q \alpha}{2 \alpha_{e x c} \pi a^{2} C} e^{-\alpha_{e x c}\left|z-v_{s} t\right|} \operatorname{sgn}(z \cdot v t)\right\} .\right.
$$

Here $\alpha_{e x c}$, which equals $4 \pi k_{\text {exc }} / \lambda_{\text {exc }}$, is the absorption coefficient at the excite wavelength, corresponding to an absorption depth $\delta=1 / \alpha_{\text {exc }}$. This solution assumes no flow of heat away from the surface. The first bracketed term in this equation represents the strain due to thermal expansion in the region near $z=0$, assuming an instantaneous temperature rise of the lattice. To be strictly valid, our calculation should take into account the rise in temperature as the nonequilibrium phonons equilibrate; however, since the phonons reach equilibrium within the first $3 \mathrm{ps}$ of the signal rise the temperature rise can be treated as instantaneous without significant impact. ${ }^{21}$ The second term in Eqn. 12 describes the acoustic pulse generated by thermal expansion propagating into the bulk at the speed of sound.

The charge in the displacement of the surface region due to expansion only is obtained by integrating Eqn. 12 from $z$ to $\infty$ over $d z^{\prime}$. To first order in $\alpha_{e x c^{z}}$, the integral is proportional to $\left(1-e^{-\alpha} e_{e x} s^{t}\right)$, which is valid in the near field region where $\alpha_{e x c^{z}}<<1$, corresponding to a probe depth that is shallow compared to the depth of excitation in the sample. This is a reasonable assumption for the experimental results presented here as the probe depth is $\approx 15 \%$ of the excite depth. We also assume that the thermally-induced expansion of the surface is fast on the time scale required for the diffusion of heat out of the surface region, hence contraction of the surface is limited by thermal diffusion. The displacement of the surface region a distance $z$ into the surface at time $t$ is thus given by

$$
\Delta u(z, t)=\left(1-e^{\left.-\alpha_{e x c} s^{l}\right)} \mathbf{T}(\mathbf{r}, t),\right.
$$

where $\mathbf{T}(\mathbf{r}, t)$ is the temperature profile due to thermal diffusion. Only diffusional flow will be considered, i.e. the mean free path of the phonons is short compared to the sample thickness. The temperature profile as a function of time and distance into the surface can be determined by solving the heat conduction equation for a point source of unit strength and an infinitely thick absorbing material 22 :

$$
\mathbf{D} \nabla^{2} \mathbf{T}(\mathbf{r}, t)=\frac{\partial \mathbf{T}(\mathbf{r}, t)}{\partial t} .
$$

The thermal diffusion constant $\mathbf{D}$ is given by $\mathbf{k} / \rho C$, where $\boldsymbol{k}$ is the thermal conductivity tensor. The initial condition used to solve Eqn. 14 is given in Eqn. 5. The three dimensional problem is simplified by separation of variables with the appropriate boundary conditions to $\mathbf{T}(\mathbf{r}, t)=\Delta T(x, y, z, t=0) T(x, t) T(y, t) T(z, t)$. On our experimental time scale the radial diffusion of heat is small compared to the Gaussian excitation spot size, thus the $x$ and $y$ Gaussian profiles can be treated as constants across the excitation spot. However, the dielectric constant is also modulated in the $y$-direction by the grating heat profile. This cannot be neglected and leads to a solution of the form

$$
T(y, l)=\frac{1}{2}\left(1+\mathrm{e}^{-D_{y} \beta 2 t} \cos (\beta y)\right),
$$


where $D_{y}$ is the diffusion constant in the $y$-direction. 23 In the $z$-direction we apply the boundary conditions $\left.\frac{\mathrm{d} T}{\mathrm{~d} x}\right|_{z=0}=0$ and
$T(z \rightarrow \infty, t)=T(t=0)$, to obtain the 1 -dimensional analytic solution 1 :

$$
\left.\left.T(z, t) \propto\left\{e^{\left(-\alpha_{e x c}\right.}{ }^{z+D\left(\alpha_{e x c}\right.}\right)^{2} t\right) \operatorname{erfc}\left(-z+\frac{2 D \alpha_{e x c} t}{2 \sqrt{D t}}\right)+e^{\left(\alpha_{e x c^{z}}+D\left(\alpha_{e x c}\right)^{2} t\right)} \operatorname{erfc}\left(z+\frac{2 D \alpha_{e x c}}{2 \sqrt{D t}}\right)\right\} .
$$

The ' mperature profile used in our calculations is thus the product of Eqns. 15 and 16. This product is substituted into Eqn. $131 \mathrm{~d}$ the net displacement of the surface, which in turn replaces the variable $\Delta \bar{n}(z, t)$ in Eqn. 4 . Equation 4 is then solved numc. xally to find the reflected diffracted signal efficiency, $\eta$.

\section{EXPERIMENTAL PROCEDURE}

A copper vapor laser amplified, colliding pulse mode-locked ring dye laser, cavity dumped at $8 \mathrm{kHz}$, was used for our experiments. 24,25 This system produced nominally $75 \mathrm{fs}$ pulses at $2.0 \mathrm{eV}$ ( $s$-polarized), which were spatial filtered to improve beam quality, then frequency doubled to $4.0 \mathrm{eV}$ (Fig. 2). The residual $2 \mathrm{eV}$ light was rotated to $p$-polarization, making all beams of like polarization, then split into two excitation beams of equal energy (maximum of $180 \mathrm{~nJ}$ each). One of the beams was directed onto a delay stage, providing control of the temporal overlap of the two excitation beams. The two beams were beams focused to a $85 \mu \mathrm{m}$ spot size, and were temporally and spatially overlapped on the sample (Fig. 1). Experiments were performed for several fringe spacings ranging from 3 to $10 \mu \mathrm{m}$. For the data presented here, the angle between the two excitation beams was $7.2^{\circ}$ as denoted by $\theta$ in Fig. 1, which led to a fringe spacing of 5 $\mu \mathrm{m}$. The excite pulses interacted with the sample to create a spatially modulated distribution of free carriers, as well as a thermal grating at the surface. The $4 \mathrm{eV}$ light was directed into a retroreflector mounted on a computer

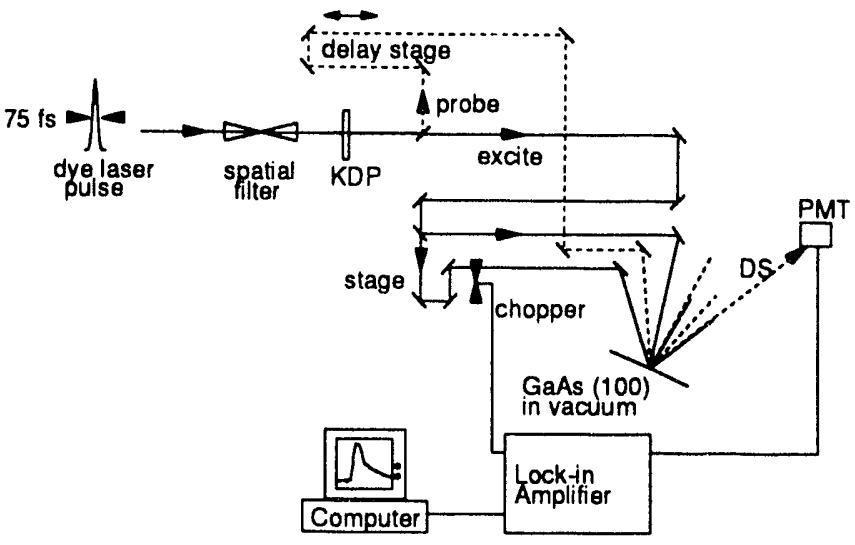

Fig. 2 Transient grating experimental setup. A 75 fs laser pulse at 620 $\mathrm{nm}$ is doubled using KDP to $310 \mathrm{~nm}$. Residual $620 \mathrm{~nm}$ light is split into two excite beams that are spatially and temporally overlapped on the GaAs surface at an angle $\theta$. The variably time delayed $310 \mathrm{~nm}$ probe is diffracted off the laser induced modulations to monitor the grating decay. The diffracted signal (DS) is detected with a photomultiplier tube (PMT) and processed via computer controlled lock-in amplification techniques.

controlled delay stage for use as the probe beam. The strongly attenuated probe was focused to a $65 \mu \mathrm{m}$ diameter on the sample, at an angle bisecting the two excitation beams as shown in Fig. 1. Because the grating depth was so shallow (i.e., $1 / \alpha_{\text {exc }} \ll \lambda_{\text {exc }}$ ), the Bragg condition for diffraction was relaxed. Hence the wave vector matching conditions for the transient grating signal were $k_{s}=k_{p} \pm \beta$, where $\beta=k_{\text {exc }}^{-k_{e x c}}$. The resulting diffracted signal appeared in reflection, collinear with both of the specularly reflected excitation beams. Excitation beam $k_{e x c}$ was chopped, and the diffracted signal collinear with the reflected excitation beam $k_{\text {ex }}^{\prime \prime}$ was detected by a photomultiplier tube and lock-in amplifier whose output was digitized by a computer as a function of defay. The geometry of the experiment was such that the detector could potentially observe both the diffracted UV probe beam and second harmonic generated by the unchopped excitation beam. However, the lock-in amplification technique used subtracted any residual competing linear or nonlinear effects from the probe or the unchopped excitation beam $k_{\text {exc }}$. In addition, the signal disappeared if any one of the three input beams were blocked, verifying it was the result of a four-wave mixing process. A power dependence study showed the diffracted signal to be linear in the intensity of each of the excitation and probe beams over one and a half orders of magnitude in signal intensity. Multiple scans of the delay line were averaged to improve signal to noise. The same baseline was obtained after each scan, demonstrating that no irreversible had damage occurred. Experiments were performed on a p-type, n-type, and undoped single crystal $\mathrm{GaAs}(100)$ samples, nominally $300-500 \mu \mathrm{m}$ thick, with a variety of dopant concentrations. The samples were placed in an ultrahigh vacuum chamber, maintained at $10^{-9}$ torr, to reduce damage due to surface contamination and condensation during low temperature experiments. All samples had a thin surface oxide layer (2-3 nm), which resulted in a slight increase in the absorption coefficient of the materials. However, because it was much thinner than the optical absorption depth it has little direct effect on the optical properties. Low temperature experiments were performed using a UHV liquid helium cooled cryostat, with a feedback temperature controller to vary the temperature. Data was taken over a range of equilibrium temperatures from 12 to $300^{\circ} \mathrm{K}$ with fluctuations of approximately $0.2^{\circ} \mathrm{K}$ over the length of a 
grating time scan. To reduce the initial temperature jump, the low temperature experiments were performed with beam energies over an order of magnitude lower than the maximum energy quoted above.

\section{RESULTS AND DISCUSSION}

When GaAs is excited by photons with energies greater than the band gap energy $(1.43 \mathrm{eV})$, electrons are excited out of the valence band into the conduction band. The three transitions allowed for a $2.0 \mathrm{eV}$ pump pulse on the $\mathrm{GaAs}(100)$ surface are s shown in Fig. 3. Electrons can be excited from the heavy hole, light hole and split-off valence bands into the conduction band $\Gamma$-valley. The creation of electron-hole pairs produces a change in the dielectric function $\tilde{\varepsilon}$, which can be described at low intensities by the Drude model since the excitation wavelength is relatively near the band edge. Single color pumpprobe measurements at $2 \mathrm{eV}$ show a rapid absorption saturation transient on the order of $100 \mathrm{fs}$ due to the electronic carriers scattering out of their initial excited state and assuming a broad energy distribution. 26 The excited distribution relaxes to the conduction band minimum via electron-phonon coupling on a 1-2 ps time scale. The relaxation of these carriers results in the transfer of heat from the carrier distribution to the lattice phonons. This creates a nonequilibrium phonon distribution which equilibrates within approximately 3 ps at $300{ }^{\circ} \mathrm{K} .{ }^{21}$ Radiative recombination brings the electrons back to the valence band within 1 ns to $1 \mu \mathrm{s}$, depending on the carrier density excited in the medium. 27

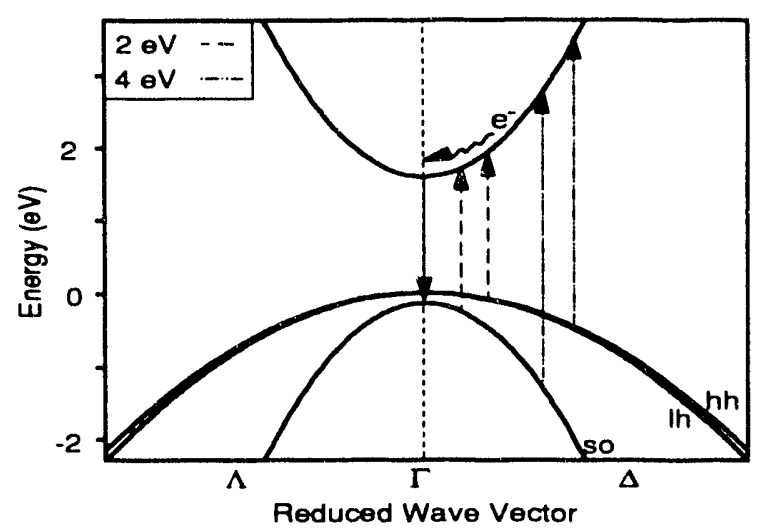

Fig. 3 Schematic of the GaAs(100) band structure. For a $2 \mathrm{eV}$ excitation transitions from the heavy hole, light hole, and split-off bands to the conduction band $\Gamma$-valley. Because the $4 \mathrm{eV}$ probe beam looks at a set of ground states further down the valence band edge than were initially excited by the $2 \mathrm{eV}$ grating, electronic effects have a negligible effect on the diffracted signal. The signal therefore arises solely from density changes at the surface created by heat transfer from the carriers to the lattice phonons and their subsequent equilibration.

Our $2 \mathrm{eV}$ excite $/ 4 \mathrm{eV}$ probe data shows no evidence of the electronic effects discussed above; instead we see a 30 33 ps rise time attributed to the thermal expansion of the GaAs perpendicular to the plane of the surface. The signal decays to the 1/e point within 150-160 ps due to conduction of heat away from the surface (Fig. 4). Since the $4 \mathrm{eV}$ light probes a set of ground states further down the valence band edge than were initially excited by the $2 \mathrm{eV}$ grating (Fig. 3), electronic effects, such as interband saturation and intervalley scattering, are negligible. Thus the diffracted signal arises sulely from density changes at the surface created by heat transfer from the carriers to the lattice phonons and their subsequent equilibration. Figure 4 shows representative data taken on n-type $\left(\mathrm{Si}, 1 \times 10^{18} \mathrm{~cm}^{-3}\right)$ and p-type $\left(\mathrm{Zn}, 6 \times 10^{18} \mathrm{~cm}^{-3}\right)$ samples at $300^{\circ} \mathrm{K}$. The signal from the n-type sample rises to a maximum within $31 \mathrm{ps,}$, and decays to the $1 / \mathrm{e}$ point within $156 \mathrm{ps}$, while the signal from the p-type sample rises in $33 \mathrm{ps}$ and decays in $150 \mathrm{ps}$. As can be seen, the differences in the rising edge and decay of the data are within the experimental noise. Only two curves are displayed for ease of viewing, however similar results were also obtained for undoped samples and different dopant concentrations. If the signal observed were due to electronic effects, a change in the optically induced carrier density, dopant type (i.e. electrons vs. holes), or dopant concentration would affect the carrier transport and thus the temporal nature of the signal. Kash and Tsang have verified this by measuring the rise and decay rate of the hot phonon population in GaAs as a function of these parameters. ${ }^{21}$ They found that the nonequilibrium decay rate in GaAs decreased as the carrier density increased, going from 8 ps to 4 ps, as the carrier density increased from $10^{16} \mathrm{~cm}^{-3}$ to $10^{17} \mathrm{~cm}^{-3}$. The nonequilibrium phonon population was also found to be much smaller in the p-type sample, because the cold (doped ) holes provided an additional mechanism for the initial cooling of the injected electrons by electron-hole scattering. This reduced the phonon lifetime to approximately $2 \mathrm{ps}$. Transient absorption studies of the effect of variations in the optically induced carrier density corroborate these results. 26,28 We did not observe a significant change in the either the rise or decay constants of our signal as the dopant type and concentration 
were changed. Nor did we observe a change in the time dependence of the signal as the excitation density was varied over an order of magnitude. We also do not observe any measurable change in the time-dependence of the signal by simultaneously rotating the polarization of the excitation pulses. Since the light and heavy hole bands are not perfectly isotropic, optical transitions from these states give rise to an anisotropic momentum distribution, the decay of which is polarization dependent. Thus an electronically derived signal should be affected by the incident light polarization. 12,29 The above results confirm that the phenomena observed is thermomechanical in nature.

The diffracted signal is composed of four components: thermal expansion of the surface and heat flow away from the surface into the bulk, and expansion and diffusion of heat in the plane of the surface. The effective time constant for the signal rise can be estimated as $\tau_{\text {eff }}=\left(\tau_{\perp}+\tau_{11}\right)^{-1 / 2} \approx 15 \mathrm{ps}$, corresponding to the $1 / \mathrm{e}$ point in the rise, where $\tau_{\perp}$ is the effective time constant for expansion perpendicular to the surface, and $\tau_{\| 1}$ is the effective time constant for expansion parallel to the surface. The individual signal contributions can be estimated as $\tau_{\|}=f / 2 v_{s}=37 \mathrm{ps}$ and $\tau_{\perp} \approx\left(\alpha_{\text {exc }} v_{s}\right)^{-1}=14 \mathrm{ps}$. The decay constant corresponding to the $1 / \mathrm{e}$ point in the decay is experimentally determined to be $156 \mathrm{ps}$. Again the contribution due to parallel thermal flow can be estimated as $D_{\|} \approx(f / 2 \pi)^{2} / D=3 \mathrm{~ns}$, and the contribution due to perpendicular flow by $D_{\perp} \approx\left(\pi / \alpha^{2} D\right)=$ $150 \mathrm{ps}$, where $D$ is the thermal diffusivity. A comparison of the individual components with the experimentally determined time constants confirms that the signal rise and fall are both dominated by the effect of phenomena occurring perpendicular to the surface. Because of the large fringe spacing used in these experiments, we expect the influence of the parallel expansion component to be negligible The mean thermal diffusion distance in GaAs is $1 \mu \mathrm{m}$, thus on the time scale of our measurements the heat will have propagated only one fifth of the way to the next grating fringe, reducing the contribution of the parallel thermal expansion to the diffracted signal to less than $5 \%$. This was verified for a range of grating fringe spacings $(3-10 \mu \mathrm{m})$. In many materials it would be possible to measure the parallel expansion of the surface. To resolve this, the grating fringe

\begin{tabular}{|c|c|c|c|c|}
\hline & \multicolumn{3}{|c|}{ Temperature } & \multirow[t]{2}{*}{ Reference } \\
\hline & $300^{\circ} \mathrm{K}$ & $50^{\circ} \mathrm{K}$ & $20^{\circ} \mathrm{K}$ & \\
\hline Bulk diffusivity, $\mathrm{cm}^{2} / \mathrm{s}$, (literature) & $0.4-1.0$ & $8-20$ & $75-188$ & $37,39,40$ \\
\hline Surface diffusivity, $\mathrm{cm}^{2} / \mathrm{s}$, (experimental) & 1.0 & 19.9 & 3.7 & This work \\
\hline Excite absorption constant, $\mathrm{cm}^{-1}$, (literature) & $4.3 \times 10^{4}$ & $\cdots$ & $\ldots$ & 30 \\
\hline Excite absorption constant, $\mathrm{cm}^{-1}$. (experimental) & $1.4 \times 10^{5}$ & $1.4 \times 10^{5}$ & $1.4 \times 10^{5}$ & This work \\
\hline Probe absorption constant, $\mathrm{cm}^{-}$(iterature) & $\times 10^{5}$ &.-- & $\cdots$ & 30 \\
\hline Velocity of sound, $\mathrm{cm} / \mathrm{s}$ & $10^{5}$ & ... & ... & 37 \\
\hline
\end{tabular}

Table 1. Parameters used to model the transient grating diffraction efficiency. (a)

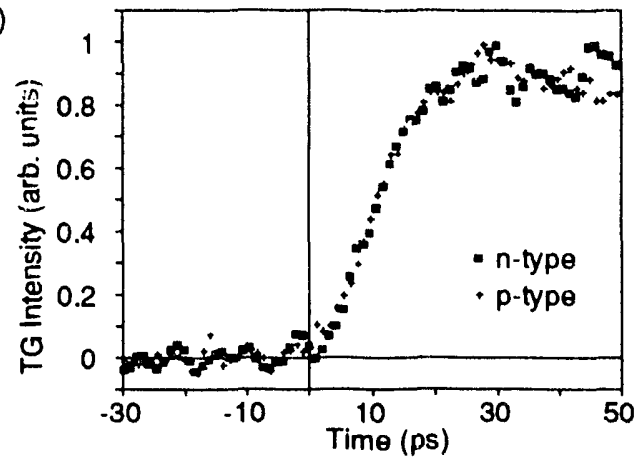

(b)

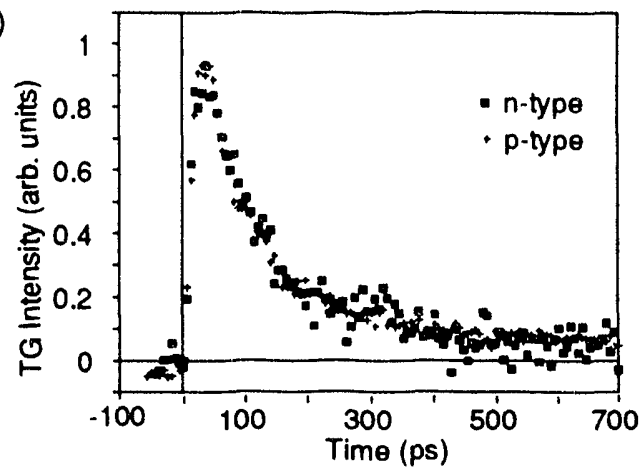

Fig. 4 Transient grating (TG) diffraction intensity versus probe delay time for data n-type (Si, $1 \times 10^{18} \mathrm{~cm}^{-3}$ ) and $\mathrm{p}-$ type $\left(\mathrm{Zn}, 6 \times 10^{18} \mathrm{~cm}^{-3}\right) \mathrm{GaAs}(100)$ samples. (a) High resolution scan of rising edge dominated by transient thermal expansion. (b) signal decay due to thermal diffusion into the bulk. Differences in data sets are within the experimental noise, verifying the signal is thermomechanical in nature.

\footnotetext{
Dashed lines indicate the $300^{\circ} \mathrm{K}$ value was used in the calculation.
} 
spacing must be on the order of or less than the skin depth of the excitation beams. In GaAs this requires a fringe spacing of $\ll 1 \mu \mathrm{m}$. This leads to a signal decay due to thermal diffusion in the plane of the sample on the order of the thermal expansion rise time, making the parallel versus perpendicular expansion difficult to resolve in GaAs. In general, this limitation is not present in all materials, and the prediction of using transient gratings to time resolve parallel versus perpendicular expansion should be possible.

Figure 5 shows a representative data set on $n$-type GaAs $\left(\mathrm{Si}, 1 \times 10^{18} \mathrm{~cm}^{-3}\right)$ with a one adjustable parameter least squares fit to the data using the model described in Sec. 2. Part (b) shows a high resolution scan of the rising edge due to thermal expansion with the same theoretical fit. Constants used in the calculation for GaAs at $300{ }^{\circ} \mathrm{K}$ are shown in Table I. The adjustable parameter in the fit is the absorption coefficient at the excitation wavelength, $\alpha_{\text {exc }}=1.5 \times 10^{5} \mathrm{~cm}^{-1}$. The calculated value of $\alpha_{\text {exc }}$ results in an optical absorption depth of $65 \mathrm{~nm}$ at the pump wavelength, while the probe penetrates to only $13 \mathrm{~nm}$ (i.e., the first 23 atomic layers of the surface. The value required to fit the data is substantially larger $\approx 4$ times) than the literature value for crystalline GaAs; however, it should be noted that there is a significant variation (a factor of at least 2) in the values reported for the absorption coefficient in the literature, due to the influence of surface preparation on this measurement. ${ }^{30}$ Also, a survey of the literature has shown that similar absorption phenomena has commonly been observed in ultrafast experiments without a definitive explanation. ${ }^{31-36}$ In order to evaluate the magnitude of the surface selectivity it is useful to define an effective grating depth for the surface component of the signal. The excitation is initially deposited with a Beer's absorption depth profile, which equilibrates with sufficient rapidity near the surface to appear uniform to the probe. The grating excitation can therefore be assumed to be uniform, i.e., $\Delta \varepsilon(z) \approx \Delta \varepsilon_{0}$, where $\Delta \varepsilon_{0}$ is a constant. The effective grating surface probe depth at the $50 \%$ absorption point can then be defined as $z_{\delta}=\lambda /\left(4 \pi \sqrt{n^{2}+k^{2}}\right) .18$ Thus the reflected diffracted signal is essentially generated within the first $6 \mathrm{~nm}$ of the surface, 1.e., the first 10 atomic layers.

(a)

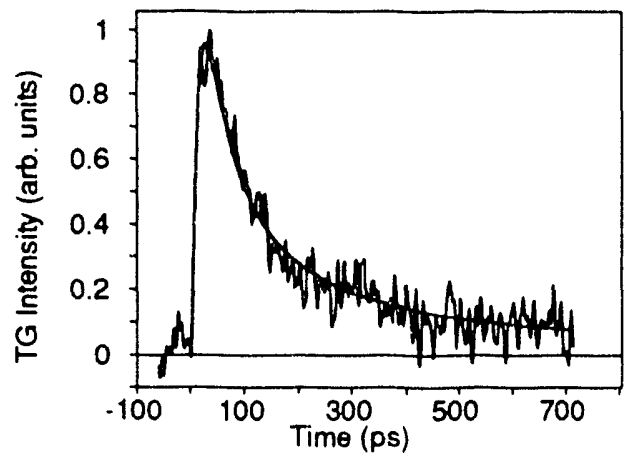

(b)

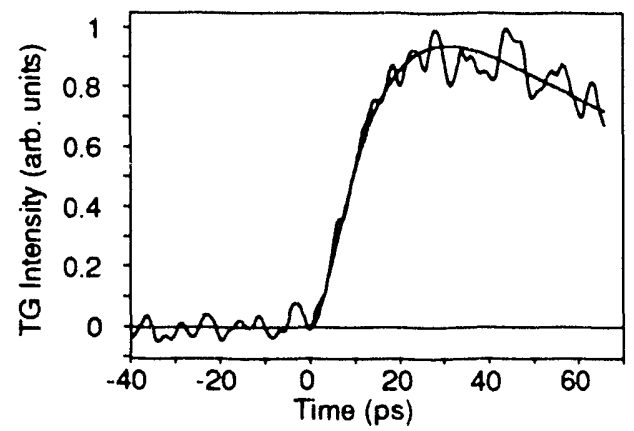

Fig. 5(a)Transient grating (TG) diffraction intensity versus probe delay time for p-type $\left(\mathrm{Zn}, 6 \times 10^{18} \mathrm{~cm}^{-3}\right) \mathrm{GaAs}(100)$ crystal at $300^{\circ}$ $K$. The smooth line through the data is a one adjustable parameter fit to the data using the model described in Sec. 2 and parameters in Table I. Adjustable parameter is $\alpha_{\text {exc }}=1.5 \times 10^{5} \mathrm{~cm}^{-1}$, the optical absorption coefficient at the excitation wavelength. (b) High resolution scan of the rising edge with the same theoretical fit.
An example of the temperature change in the grating peaks as a function of time after initial excitation and depth into the surface is shown in Fig. 6. An estimate of the average temperature

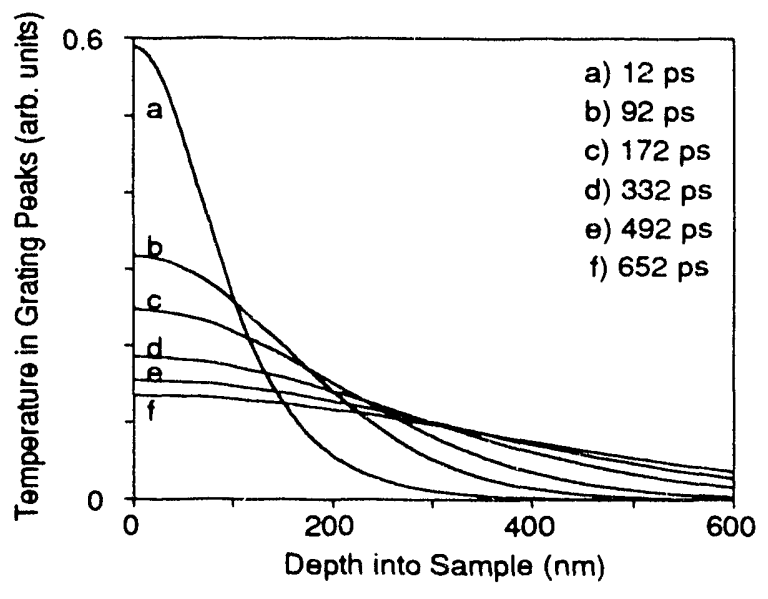

Fig. 6 Spatial thermal profiles used to calculate the fit in Fig. 5 . lncrease in temperature above the equilibrium value in the grating peaks is plotted versus absorption depth in the sample. The six zurves correspond to delay times of $12,92,172,332,492$, and 652 ps after the initial thermal excitation. 
rise of the lattice at room temperature associated with the thermal expansion signal, is given by $\Delta T=(E / A \delta \rho C) \approx 22-220$ ${ }^{\circ} \mathrm{K}$. The temperature range : iven is based on the range of laser energies densities $\left(0.4-4.0 \mathrm{~mJ} / \mathrm{cm}^{2}\right)$ used in our experiments. Here $E$ is the absorbed laser energy (18-180 nJ/beam), corrected for the normal incidence reflectivity of the surface $(1-R=$ $66 \%$ ). ${ }^{30}$ The excitation spot size is $A=64 \times 10^{-6} \mathrm{~cm}^{2}$, and $\delta=65 \mathrm{~nm}$ is the experimentally determined value for the optical absorption depth at the excite wavelength. The room temperature specific heat at constant pressure per unit volume is $\rho C=$ $1.8 \mathrm{~J}^{\circ} \mathrm{K} / \mathrm{cm}^{3} .37$ If the literature value for the optical absorption depth is used, this temperature range reduces to $5.8-58{ }^{\circ} \mathrm{K}$. The maximum temperature value corresponds to a maximum net perpendicular displacement of the surface of $(T \gamma \delta) \approx 1 \AA$, where $\gamma=6 \times 10^{-6} / \mathrm{K}^{\circ}$ is the linear expansion coefficient for bulk GaAs. ${ }^{38}$ Assuming this displaccment is uniformly distributed throughout the optical excitation absorption depth, this corresponds to an average displacement per atomic layer of $(1 \AA / 115$ atomic layers $)=0.008 \AA$, or $0.1 \%$. It is the sum of these individual displacements which gives rise to the overall net displacement of $1 \AA$. This is of course an average estimate, as the surface atomic layers are expected to have a displacement several times larger than those 115 layers into the bulk.

\section{4.a. Temperature dependent surface thermal diffusivity}

Figure 7 shows data taken on a p-type sample with a $\mathrm{Zn}$ dopant concentration of $6 \times 10^{18} \mathrm{~cm}^{-3}$ at 300,50 , and $20^{\circ} \mathrm{K}$. This sample was chosen because literature values exist for the bulk diffusivity as a function of temperature for GaAs with this dopant type and concentration. ${ }^{39}$ The solid lines through the data are fits using the model in Sec. 2 with the parameters appropriately adjusted for temperature (see Table I). The material band gap energy increases approximately 35 $\mathrm{meV}$ as the temperature decreases to several degrees Kelvin. However, because the excite wavelength is approximately 500 $\mathrm{meV}$ above the band edge, the effective absorption is not expected to change significantly as a function of temperature. The low temperature data is fit using the value of $\alpha_{\text {exc }}$ obtained at room temperature, and varying the diffusivity until a reasonable match with the data was obtained. The rising edge becomes faster ( $33 \mathrm{ps} \rightarrow 25 \mathrm{ps)}$ as the temperature decreases

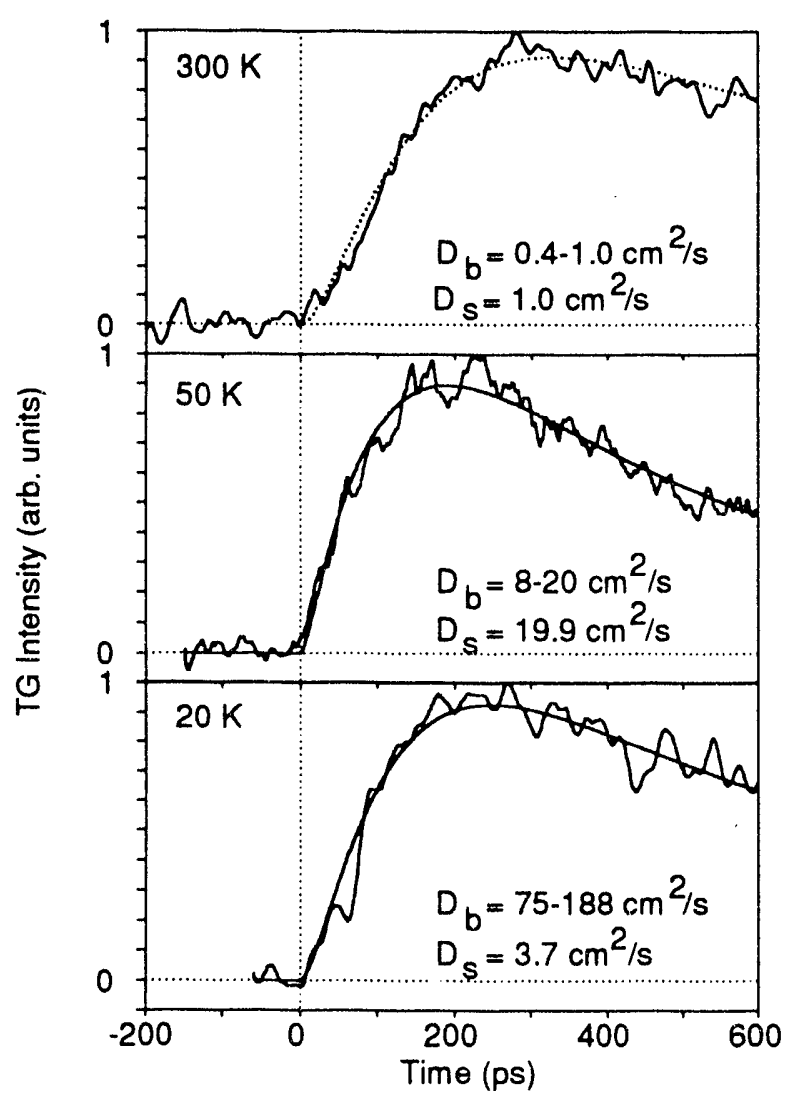

Fig. 7 Transient grating (TG) diffraction intensity is versus probe delay time is plotted for p-type $\left(\mathrm{Zn}, 6 \times 10^{18} \mathrm{~cm}^{-3}\right) \mathrm{GaAs}(100)$ at 20 , 50, and $300^{\circ} \mathrm{K}$. The smooth lines through the data are theoretical fits using the model described in Sec. 2 and parameters in Table I. to 50 ' $\mathrm{K}$, but begins to slow down again by $20^{\circ} \mathrm{K}$. This is consistent with the fact that the linear expansion coefficient becomes negative between 12 and $56{ }^{\circ} \mathrm{K}$. corresponding to a region of contraction rather than expansion. 38

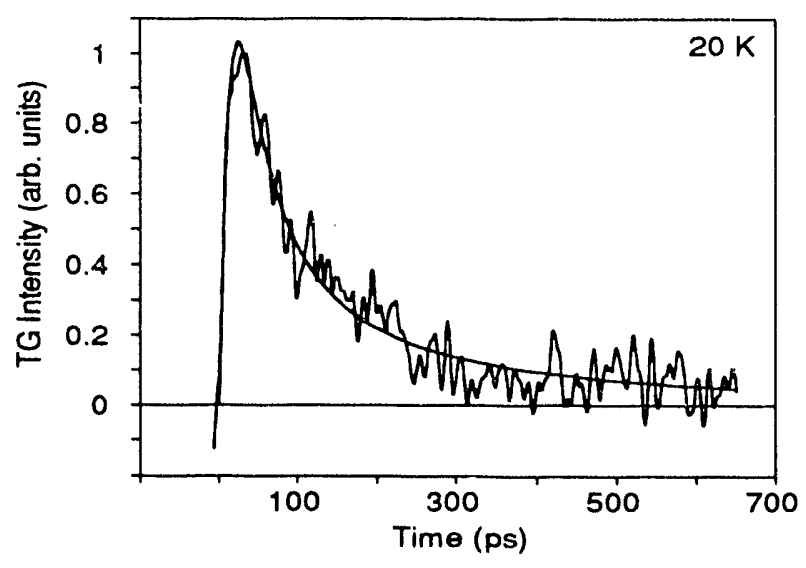

Fig. 8 Transient grating (TG) diffraction intensity versus probe delay time for p-type $\left(\mathrm{Zn}, 6 \times 10^{18} \mathrm{~cm}^{-3}\right) \mathrm{GaAs}(100)$ at $20^{\circ} \mathrm{K}$. The smooth line through the data is a theoretical fit using the model described in Sec. 2 and parameters in Table 1 . 
theoretical model. One possibility for the anomaly is the assumption in Eqn. 13 that the diffusion of heat out of the surface region is slow on the time scale of the expansion signal. While this is a valid assumption to first order, the two phenomena are interrelated. Changing the value of $\alpha_{\text {exc }}$ primarily influences the rising edge, while the falling ejge is dependent on both $D_{s}$ and $\alpha_{e x c}$, thus both phenomena must ve treated simultaneously. The effect of a nonequilibrium temperature distribution can be included by replacing the standard heat conduction equation (Eqn. 14) with the coupled nonlincar equations relating the electron and phonon temperature distributions. 42 One final consideration is that our model is based upon equations of motion for the bulk crystal. The force constants of atoms near the surface are assumed to be the same as for those in the bulk, and thus are evaluated with respect to the positions corresponding to uniform thermal expansion throughout the crystal. In reality, the mean-square amplitudes of atoms at the surface of the crystal are significantly larger than in the bulk. Thus in a more general treatment of surface phenomena anharmonic effects should be taken into consideration.

\section{CONCLUSIONS}

We have directly measured the transient thermal expansion and diffusivity of a GaAs surface using a two-color reflection transient grating technique. For a highly absorbing material, such as GaAs, the technique is selective to the first few atomic layers of the surface. Isolation of the expansion signal requires the selection of wavelengths which pump and probe different regions of the electronic band structure in the sample. The surface thermal diffusivity was determined to be equivalent to the bulk value above $50^{\circ} \mathrm{K}$ : however, below $50^{\circ} \mathrm{K} D_{s}$ was over an order of magnitude slower than the bulk diffusivity due to increased phonon boundary scattering. This reduction in the surface diffusivity may have implications in the low temperature performance of thin film semiconductor devices. We have demonstrated that the expansion signal perpendicular to the surface can be successfully modeled using a straightforward one dimensional analytic theory. The work reported here is restricted to large grating fringe spacing, validating the applicability of this technique to measure the perpendicular surface thermal expansion and diffusivity. However, the true power of the reflection transient grating technique lies in its ability to simultaneously measure phenomena in and out of the plane of the surface by varying the fringe spacing of the grating. Thus this promises to be the first technique with the capability of time resolving both the parallel and perpendicular thermal expansion and diffusivity of a surface.

\section{ACKNOWLEDGMENTS}

We would like to thank C. D. Marshall for many useful discussions. This work was supported by a grant from the Office of Naval Research. We also wish to acknowledge the U. S. Dept. of Energy, Office of Basic Energy Sciences, Chemical Sciences Division under Contract No. DE-AC03-76SF00098 for specialized equipment used in these experiments. D. M. P. acknowledges additional support from the U. S. Department of Energy and Lawrence Livermore National Laboratory under Contract No. W-7405-Eng-48.

\section{REFERENCES}

1. J. H. Bechtel, "Heating of solid targets with laser pulses," J. Appl. Phys., vol. 46, 1975, pp. $1585-1593$.

2. M. C. Downer, C. V. Shank, "Ultrafast heating of silicon on sapphire by femtosecond optical pulses," Phys. Rev. Lett., vol. 56, 1986, pp. 761-764.

3. J. G. Fujimoto, J. M. Liu, E. P. Ippen, N. Bloembergen, "Femtosecond laser interaction with metallic tungsten and nonequilibrium electron and lattice temperatures," Phys. Rev. Lett., vol. 53, 1984, pp. 1837-1840.

4. G. L. Eesley, "Generation of nonequilibrium electron and lattice temperatures in copper by picosecond laser pulses," Phys. Rev. B, vol. 33, 1986, pp. 2144-2151.

5. C. Thomsen, H. T. Grahn, H. J. Maris, J. Tauc, "Surface generation and detection of phonons by picosecond light pulses," Phys. Rev. B, vol. 34, 1986, pp. 4129-4138.

6. C. D. Marshall, I. M. Fishman, R. C. Dorfman, C. B. Eom, M. D. Fayer, "Thermal diffusion, interfacial thermal barrier, and ultrasonic propagation in $\mathrm{YBa}_{2} \mathrm{Cu}_{3} \mathrm{O}_{7-x}$ thin films: surface selective transient grating experiments," Phys. Rev. B, in press.

7. C. D. Marshall, I. M. Fishman, A. Tokmakoff, C. B. Eom, J. M. Phillips, M. D. Fayer., "Thermal boundary resistance and diffusion measurements on thin $\mathrm{YBa}_{2} \mathrm{Cu}_{3} \mathrm{O}_{7-\mathrm{x}}$ films with $\mathrm{MgO}$ and $\mathrm{SrTiO} 3$ substrates using the transient grating method," J. Appl. Phys., in press. 
8. J. E. Rothenberg, "Observation of the transient expansion of heated surfaces by picosecond photothermal deflection spectroscopy," Opt. Lett., vol. 13, 1988, pp. 713-715.

9. D. M. Pennington, C. B. Harris, Tech. Dig., Annual Meti. Op. Soc. Amer San, Jose, CA, 1991, paper ThDD2.

10. M. D. Fayer, "Picosecond holographic grating generation of ultrasonic waves," IEEE J. Quantum Electron., vol. QE-22. 1986. pp. 1437-1452.

11. K. A. Nelson, R. Casalegno, R. J. D. Miller, M. D. rayer, "Laser-induced excited state and ultrasonic wave gratings: Amplitude and phase grating contributions to diffraction," J. Chem. Phys., vol. 77, 1982, pp. 1144-1152.

12. A. L. Smirl, T. F. Boggess, B. S. Wherrett, G. P. Perryman, A. Miller, "Picosecond transient orientational and concentration gratings in germanium," IEEE J. Quantum Electron., vol. QE-19, 1983, pp. 690-700.

13. J. R. Andrews, R. M. Hochstrasser, "Transient grating studies of energy deposition in radiationless processes," Chem. Phys. Lett., vol. 76, 1980, pp. 207-211.

14. H. J. Eichler, P. Gunter, D. W. Pohl, Laser-induced dynamic gratings. Berlin: Springer-Verlag, 1986.

15. J. S. Meth, C. D. Marshall, M. D. Fayer, "Experimental and theoretical analysis of transient grating generation and detection of acoustic waveguide modes in ultrathin solids," J. Appl. Phys., vol. 67, 1990, pp. 3362-3377.

16. D. M. Pennington, C. B. Harris, "Dynamics ofphotothermal surface expansion and diffusivity using laser-induced holographic gratings," IEEE J. Quantum Electron., vol. 28, 1992, pp. 2523-2534.

17. I. M. Fishman, C. T!. Marshall, J. S. Meth, N. D. Fayer, "Surface selectivity in four-wave mixing: transient gratings as a theoretical an , experimental example," J. Opt. Soc. Am. B, vol. 8, 199 ,pp. 1880-1888.

18. C. D. Marshail, Ph.D. dissertation. Stanford University, 1992.

19. I. M. Fishman, C. D. Marshall, A. Tokmakoff, M. D. Fayer, "Transient grating diffraction from an interface between two materials: theory and experimental application," J. Opt. Soc. Am. B, in press.

20. k. C. Desai, M. D. Levenson, J. A. Barker, "Forced Rayle:gh scattering: thermal and acoustic effects in phaseconjugate wave-fron: generation," Phys. Rev. A, vol. 27, 1983, pp. 1968-1976.

21. J. A. Kash. J. C. Tsang, "Secondary emission studies of hot carrier relaxation in polar semiconductors," Solid State Electron., vol. 31, 1988, pp. 419-424.

22. H. S. Carslaw, J. C. Jaeger, Conduction of heat in solids. London: Oxford University Press, 1959.

23. M. D. Fayer, "Exciton Cohere- e," in Spectroscopy and Excitation Dynamics of Condensed Molecular Systems, V. M. Agranovitch, R. M. Hochstrasser, Eds., 1983.

24. R. L. Fork. B. I. Greene, C. V. Shank, "Generation of optical pulses shorter than $0.1 \mathrm{psec}$ by colliding pulse mode locking," Appl. Phys. Lett., vol. 38, 1981, pp. 671-672.

25. W. H. Knox, M. C. Downer, R. L. Fork, C. V. Shank, "Amplified femtosecond optical pulses and continuum generation at $5 \mathrm{kHz}$ repetition rate," Opt. Lett., vol. 9, 1984, pp. 552-554.

26. W. Lin, R. W. Schoenlein. J. G. Fujimoto, E. P. Ippen, "Femtosecond absorption saturation studies of hot carriers in GaAs and AIGaAs," IEEE J. Quantum Electron., vol. 24, 1988, pp. 267-275.

27. W. B. Wang, N. Ockman, M. Yan, R. R. Alfano, "The intervalley $C_{6}$ to $G_{6}, L_{6}$ scattering time in GaAs measured by ultrafast pump-probe infrared absorption spectroscopy," Solid-State Electron., vol. 32, 1989, pp. 1337-1345.

28. T. Gong, W. L. Nighan, P 1. Fauchet, "Hot-carrier Coulomb effect in GaAs investicated by femtosecond spectroscopy around the band edge," Appl. Phys. Lett., vol. 57, 1990, pp. 2713-2715.

29. J. L. Oudar, A. Migus, D. Hulin, G. Grillon, J. Etchepare, A. Antonetti, "Femtosecond orientational releaxtion of photoexcited carriers in GaAs," Phys. Rev. Lett., vol. 53, 1984, pp. 384-387.

30. D. E. Aspnes, A. A. Studna, "Dielectric functions and optical parameters of Si, Ge, GaP, GaAs, GaSb, InP, InAs, and InSb from 1.5 to $6.0 \mathrm{eV}$," Phys. Rev. B, vol. 27, 1983, pp. 985-1009.

31. C. V. Shank, R. Yen, C. Hirlimann, "Femtosecond time-resolved surface structural dynamics of optically excited silicon," Phys. Rev. Lell., vol. 51, 1983, pp. 900-902.

32. P. Saeta, J. K. Wang, Y. Siegal, N. Bloembergen, E. Mazur, "Ultrafast electronic disordering during femtosecond laser melting of GaAs," Phys. Rev. Lett., vol. 67, 1991, pp. 1023-1026.

33. J. M. Liu, A. M. Malvezzi, N. Bloembergen, "Picosecond laser melting and evaporation of GaAs surfaces," Appl. Phys. Lett., vol. 49, 1986, pp. 622-624.

34. N. Fabricius, P. Jermes, D. von der Linde, P. Pospieszczyk, B. Stritzker, "Observation of superheating during picosecond laser melting," Solid State Commun., vol. 58, 1986, pp. 239-242.

35. J. M. Moison, F. Barthe, M. Bensoussan, "Laser-induced nonlinear absorption in silicon: Free-carrier absorption versus thermal effects," Phys. Rev. B, vol. 27, 1983, pp. 3611-3619.

36. A. Aydinli, H. W. Lo, M. C. Lee, A. Compann, "Induced absorption in silicon under intense laser excitation: Evidence for a self-confined plasma," Phys. Rev. Lett., vol. 46, 1981. pp. 1640-1643. 
37. R. Blachnik, W. inress, O. Madelung, in Landolt-Bornstein New Series, Vol. III/17a, O. Madelung, Eds., 1982, pp. 234-248, 543-545, and references therein.

38. J. S. Blakemore, "Semiconducting and other major properties of gallium arsenide," J. Appl. Phys., vol. 53, 1982, pp. R123-R181.

39. M. G. Holland, "Phonon scattering in doped GaAs from magnetothermal conductivity studies," in Proc. 7th Int. Conf. Phys. Semiconductors, Eds., 1964, pp. 713-717.

40. O. Madelung, in Landolt Bornstein New Series, Vol. III/22a, O. Madelung, Eds., 1987, pp. 88, 113, and references therein.

41. P. Carruthers, "Scattering of phonons by elastic strain fields and the thermal resistance of dislocations," Phys. Rev., vol. 114, 1959, pp. 995-1001.

42. S. I. Anisimov, B. L. Kapeliovich, T. L. Perel'man, "Electron emission from metal surfaces exposed to ultrashort laser pulses," Zh. Eksp. Teor. Fiz., vol. 66, 1974, pp. 776-781.

43. R. M. More, K. H. Warren, D. A. Young, G. B. Zimmerman, "A new quotidian equation of state (QEOS) for hot dense matter," Phys. Fluids, vol. 31, 1988, pp. 3059-3078.

44. X. Y. Wang, M. C. Downer, "Femtosecond time-resolved reflectivity of hydrodynamically expanding metal surfaces," Opt. Lett., vol. 17, 1992, pp. 1450-1452.

45. F. A. Lindemann, "The calculation of molecular vibration frequencies," Phys. Z., vol. 1 pp. 609-612 1910. 

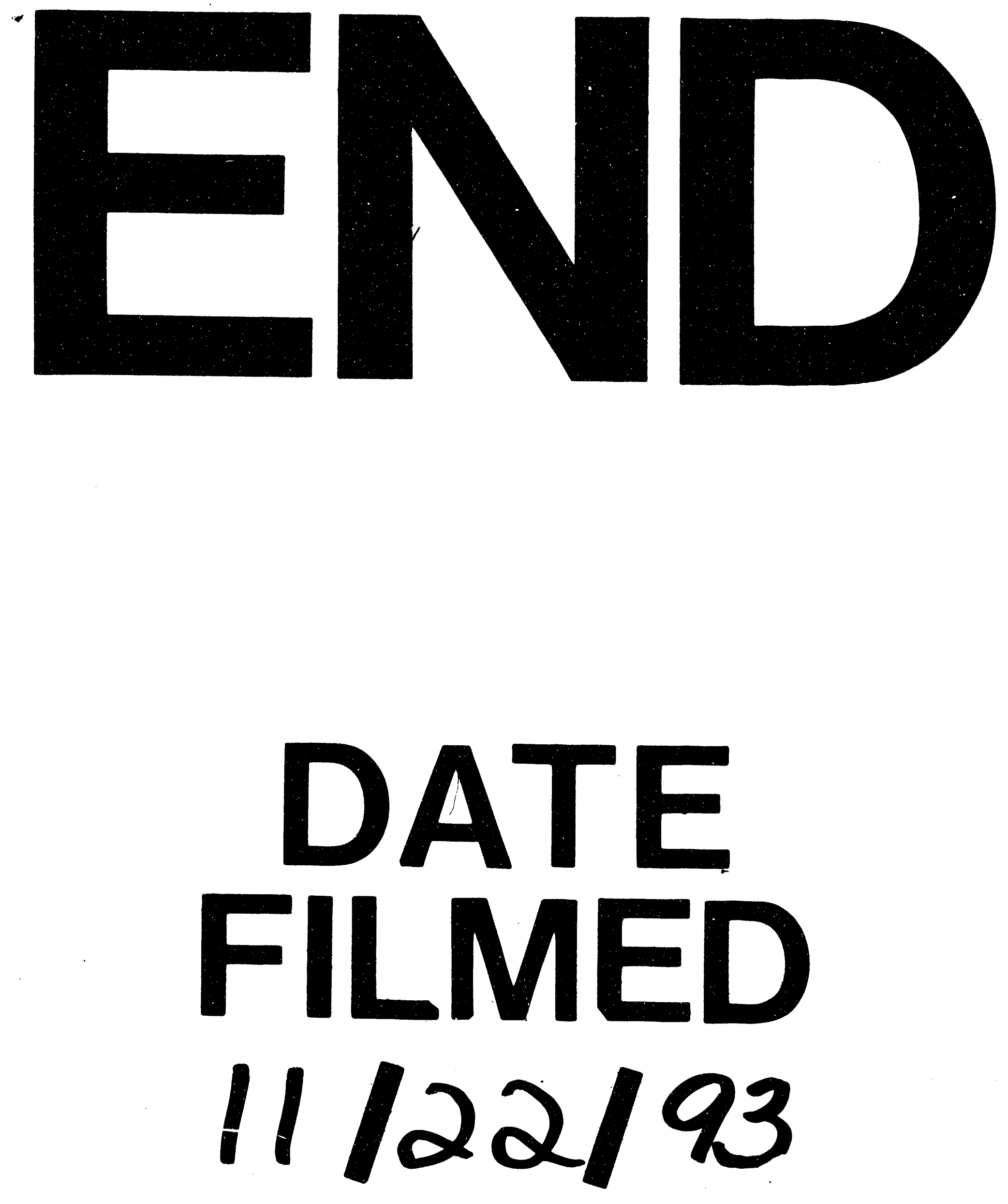
$\longrightarrow$ 